\title{
Research Paper \\ Effect of Six Weeks of Progressive Jump-Landing Training on Jump Shooting Ac- curacy and Knee Valgus Angle in Male Basketball Players with Dynamic Knee Valgus
}

\author{
"Saeed Ghobadi Nezhad ${ }^{1}{ }^{\circ}$, Seyyed Hosein Hoseini ${ }^{2}$, Ali Asghar Norasteh ${ }^{1}$ (두
}

1. Department of Sport Injuries and Corrective Exercises, Faculty of Physical Education and Sport Sciences, University of Guilan, Rasht, Guilan, Iran. 2. Department of Physical Education and Sport Sciences, Faculty of Physical Education and Sport Sciences, University of Guilan, Rasht, Guilan, Iran.

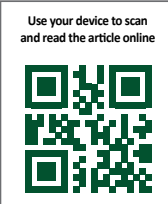

CItration: Ghobadi Nezhad S, Hoseini SH, Norasteh AA. [Effect of Six Weeks of Progressive Jump-Landing Training on Jump Shooting Accuracy and Knee Valgus Angle in Male Basketball Players with Dynamic Knee Valgus (Persian)]. Journal of Sport Biomechanics. 2021; 7(1):148-163. https://doi.org/10.32598/biomechanics.7.2.6

https://doi.org/10.32598/biomechanics.7.2.6

Keywords: Progressive jumplanding training, Jump shooting, Dynamic knee valgus, basketball players

\section{A B STRACT}

Objective Dynamic Knee Valgus (DKV) causes many acute and chronic knee injuries during deceleration and landing in sports activities and can affect the athletes' specific skills. This study aims to investigate the effect of 6 weeks of progressive jump-landing training on jump shooting accuracy and knee valgus angle in male basketball players with DKV.

Methods This is a quasi-experimental study conducted on 24 male basketball players with DKV in Mazandaran, who were selected purposefully. They were randomly divided into training and control groups. Their jump shooting accuracy was assessed by Boddington's Basketball Jump Shooting Accuracy Test, and their DKV angle was measured by a two-dimensional evaluation method in Quinoa software. In addition to their routine exercises, the training group performed the jump-landing training for 6 weeks at 3 sessions per week, while the control group performed their usual exercises only. In order to compare the two study groups, ANCOVA was used.

Results The progressive jump-landing program had a significant effect on jump shooting accuracy $(P=0.001)$ and $D K V$ angle $(P=0.001)$.

Conclusion Six weeks of progressive jump-landing training can improve the jump shooting accuracy and knee valgus angle in basketball players with DKV. Therefore, jump landing exercises are recommended for improving the jump shooting skill and knee alignment in these athletes.

\section{Extended Abstract}

\section{Introduction}

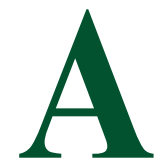

nterior cruciate ligament (ACL) injury is the most common knee ligament injury, the mechanism of which is often in noncollision events such as jump-landing, cutting, and deceleration [1]. Basketball is inherently a vertical sport and requires 35-46 jump and landing activities per game. The multidirectional nature of basketball requires constant acceleration and deceleration, forcing athletes to change direction every 2-3 seconds. When landing, the lower limbs are in certain position where the trunk has anterior flexion, hip adduction and internal rotation, 20-30 degree knee flexion, knee valgus, tibia external rotation and pronation of the anterior aspect of the foot, which can lead to complete rupture of the ACL [3].

\section{* Corresponding Author:}

Saeed Ghobadi Nezhad

Address: Department of Sport Injuries and Corrective Exercises, Faculty of Physical Education and Sport Sciences, University of Guilan, Rasht, Guilan, Iran. Tel: +98 (938) 7889494

E-mail: saeedqobadii@gmail.com 
If the basketball player's lower limbs be in good condition while shooting, the player has the ability to control the body and balance after successive attacks so that $\mathrm{s} /$ he can use the opponent's defensive gaps to shoot. Moreover, improper alignment after the shot, especially in rebounds, is a risk factor for lower limb injuries, especially the ACL $[5,6]$. In most studies, the athletes that are at risk for non-collision ACL injury have not been investigated, and have mostly used expensive biomechanical tools. This study aims to investigate the effect of 6 weeks of progressive jump-landing training on jump shoot accuracy and knee valgus angle of basketball players with Dynamic Knee Valgus (DKV).

\section{Methods}

This is a quasi-experimental and interventional study with a pre-test/post-test design. The study population consists of all male basketball players with DKV aged 16-20 year in Mazandaran, Iran who had at least 4 years of regular exercise, three times per week. The number of samples was estimated 24 using $\mathrm{G}$ power software, considering $\mathrm{r} 2=0.50$, test power $=0.80$ and alpha $=0.05$ according to previous studies $[10,11]$. Subjects were randomly divided into two training and control groups. The training group, in addition to their usual exercises, performed the jump-landing training for 6 weeks, 3 sessions per week, while the control group performed their routine exercises only. After obtaining a written informed consent from the participants, their height and weight were measured using a tape measure and a scale. In order to identify people with DKV, the double-leg Squat test was used. Subjects' shooting skills were assessed using Boddington's Basketball Jump Shooting Accuracy Test, and DKV angle was measured using a camera (Nikon D3300, Thailand) and Quinoa software at two sessions.

\section{Results}

Table 1 shows the demographic characteristics of the two groups. The results showed that in the post-test phase, the jump shooting accuracy of the training group was significantly higher compared to the control group $(\mathrm{P}=0.001)$ while the DKV angle of the training group was significantly lower compared to the control group $(\mathrm{P}=0.001)$. Therefore, it can be said that jump-landing training had a significant effect on these variables.

\section{Discussion and Conclusion}

The purpose of the present study was to evaluate the effectiveness of 6 weeks of progressive jump-landing training on jump shooting accuracy and knee valgus angle of basketball players with DKV . The results showed that after the training protocol, significant changes were made in the accuracy of jump shooting accuracy and DKV angle. One of the possible reasons for the effectiveness of the program is that the subjects received the necessary instructions and verbal feedback to improve the kinematics of the lower limb, especially the correction of the knee alignment in the frontal plane. Hence, the subjects performed the landing task with better skill after completing the training period. In terms of jump shooting accuracy, the program improved the player's ability to stop dribbling and change dynamic horizontal displacement into vertical displacement for shooting, in addition to transferring the force from the lower limbs to the trunk and upper limbs. This causes the player to be at the highest point and away from the opponent's defenders while jumping to shoot, leading to be more successful in scoring. On the other hand, it is believed that plyometric exercises increase the ability to use maximum force in the shortest possible time, stimulate the rapid change of eccentric contraction to concentric contraction, and allow more work to be done in less time. By using the elastic properties of the muscle-tendon unit, these exercises increase the power and, as a result, performance during functional activities [28].

Table 1. Demographic characteristics of the participants

\begin{tabular}{|c|c|c|c|}
\hline \multirow{2}{*}{ Variable } & \multicolumn{2}{|c|}{ Mean $\pm S D$} & \multirow{2}{*}{$\mathbf{P}$} \\
\hline & Training $(n=12)$ & Control (n=12) & \\
\hline Age (year) & $17.58 \pm 1.17$ & $17.92 \pm 1.5$ & 7.0 \\
\hline Height (cm) & $191.33 \pm 5.08$ & $192.67 \pm 4.49$ & 0.5 \\
\hline Weight (kg) & $86.92 \pm 3.42$ & $87.33 \pm 5.29$ & 0.22 \\
\hline Body Mass Index & $23.75 \pm 0.81$ & $23.5 \pm 0.69$ & 0.57 \\
\hline
\end{tabular}




\section{Ethical Considerations}

Compliance with ethical guidelines

This study obtained its ethical approval from the Sport Sciences Research Institute of Iran (Code: IR.SSRI. REC.1399.899).

Funding

This study was extracted from the MA. thesis of first author at the Department of Sport Injuries and Corrective Exercises, Faculty of Physical Education and Sport Sciences, University of Guilan, Rasht.

Authors' contributions

All authors contributed equally in preparing this article

Conflicts of interest

The authors declared no conflict of interest. 
مقاله يزووهشى

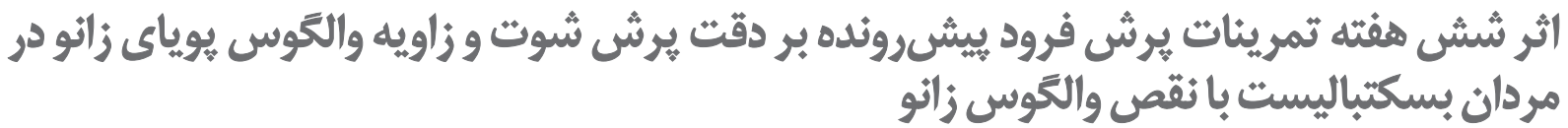

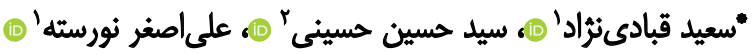
1. كروه آسيبشناسى ورزشى و حركات اصلاحى، دانشكده تربيتبلدى و علوم ورزشى، دانشعاه كيلان، رشت، كيلان، ايران.

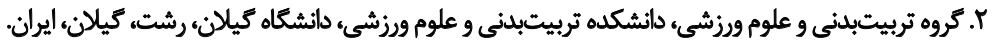

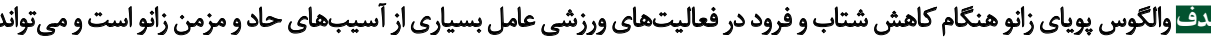

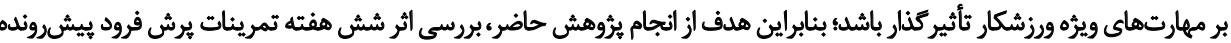

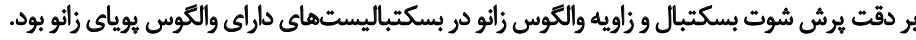

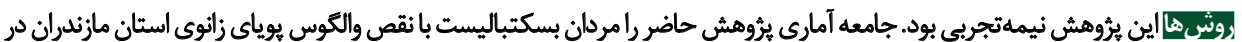

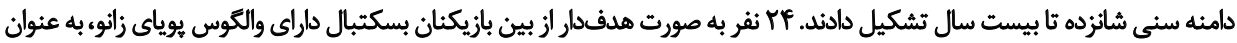

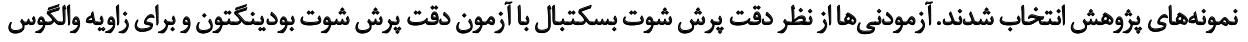

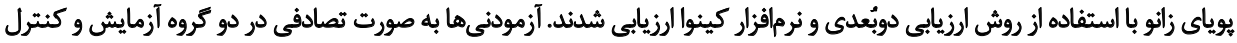

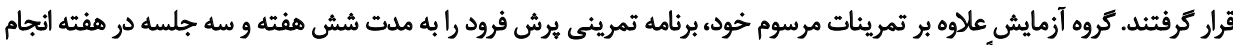

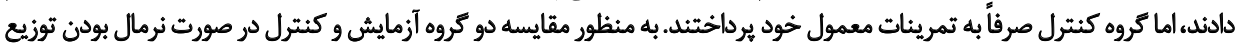

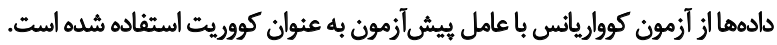

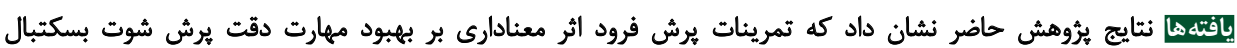

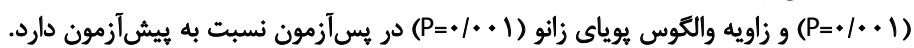

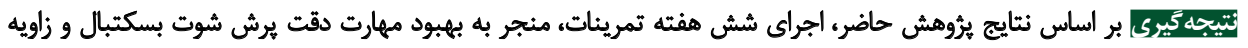

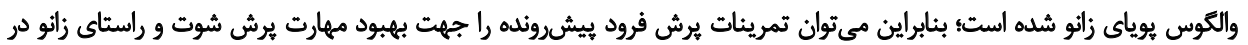

كليدوازهها:

تمرينات برش فرون برون

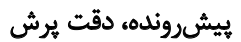

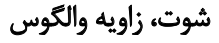
بويا زانو، بازيكنان بسكتبال

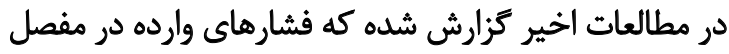

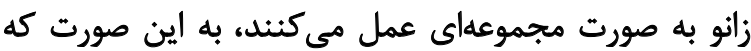

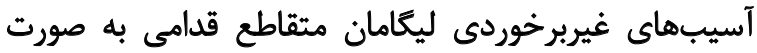

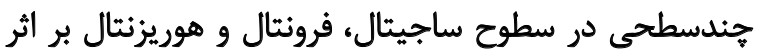
افزايش حركت و فشار اتفاق مىافتد [r].

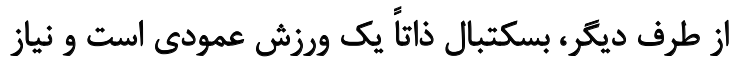

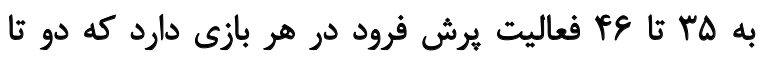

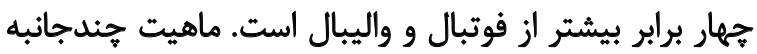

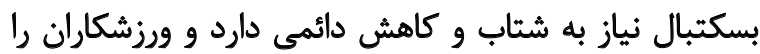
مجبور مى كند هر دو تا سه ثانيه تغيير جهت بائهى بدهند.

آسيب ليكامان متقاطع قدامى شايعترين آسيب ليكامانى

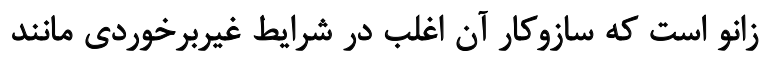

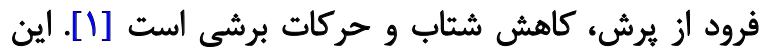

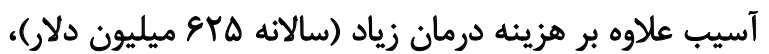

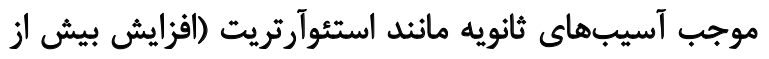

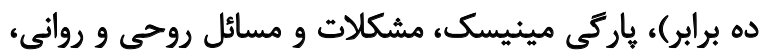

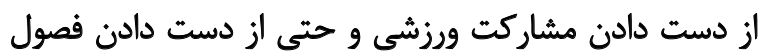
ورزشى مىشود. 


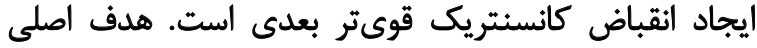

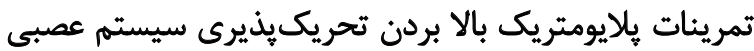
جهت بهبود توانايى واكنشى سيستم عصبى عضلانى است. اصول اساسى روش تمرين به سبك يلايومتريك عبارت است

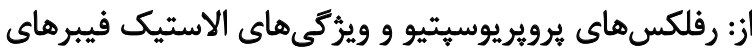

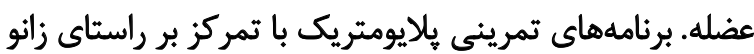

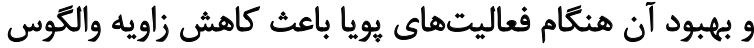
زانو و نيروهاى عكسالعمل زمين مي في فئمالي باعث كاهثر در اين خصوص هرينكتون با اعمال ههار هفته تمرينات

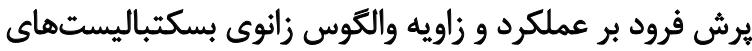

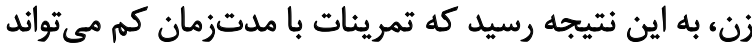

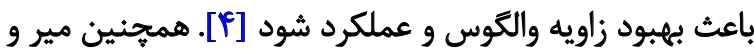

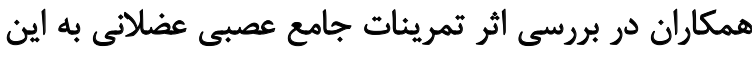

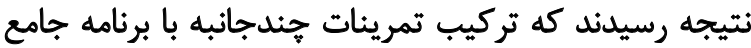

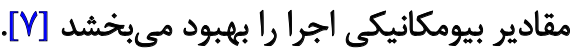

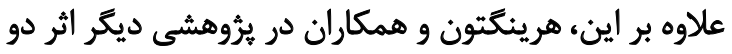

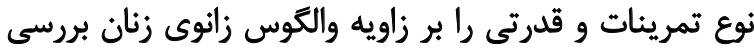

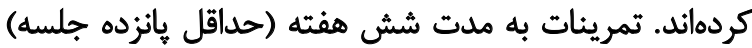

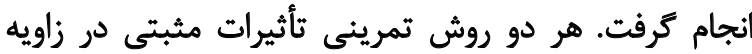
والكوس زانو در بعضى آزمونها و نه دمر همه آنها داشتند. تمرينات قدرتى باعث ايجاد تغييرات جشمكير در زاويه

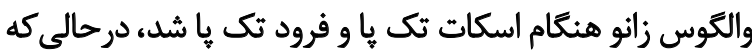

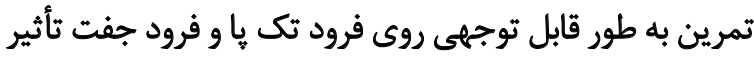
داشت [1]].

همجنين احمد السيد احمد، يروهشى تحت عنوان

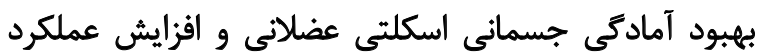

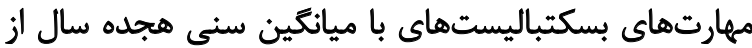

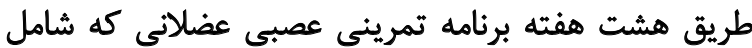

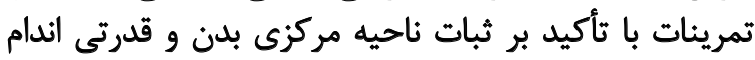

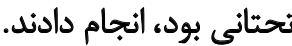

كروه تمرينى عصبى عضلانى در آزمونهاى مهارتى ايفرد

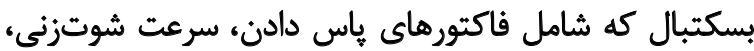

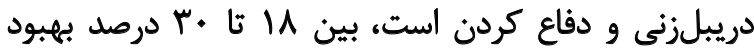

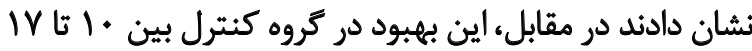
درصد بود [9]. حسين محمود و مونيم الوسوفى در بيروهشى اثر تمرينات

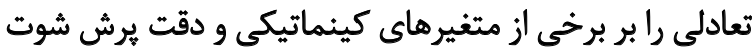

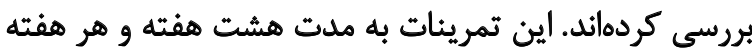

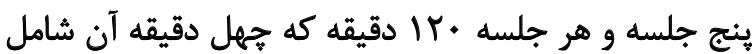
تمرينات تعادلى مىشد، صورت كرفت. نتايج بيانكَ بهبود در مسير حركت مركز ثقل براى مسيرهاى

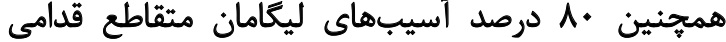

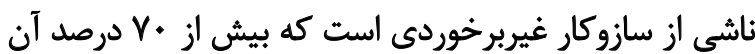

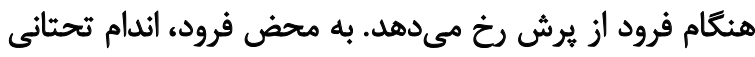

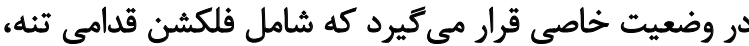

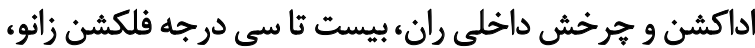

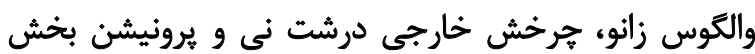

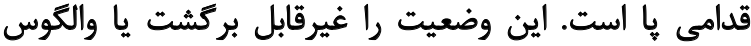

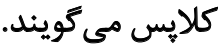

فرود با وضعيت والكوس كلايس موجب اعمال مقدار زيادى

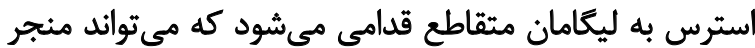

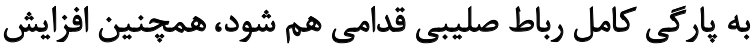

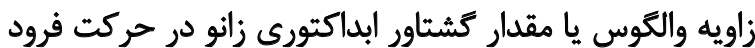

از يرش با آسيبهاى زانو ارتباط دارد [باري. وضعيث والكوس يوياى زانو به عنوان تركيبى از آداكثن و

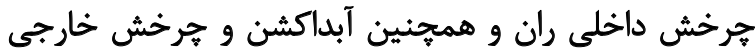

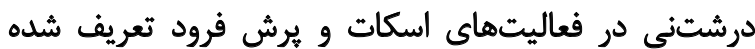

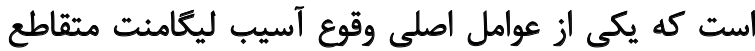

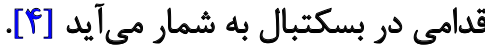
علاوه بر اين، اكر هنكام شوت زدن، اندام تحتانى بسكتباليست

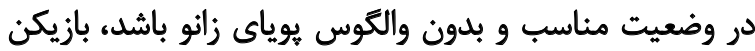

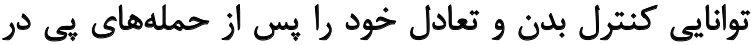

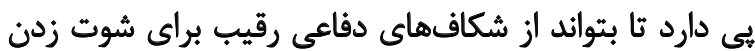

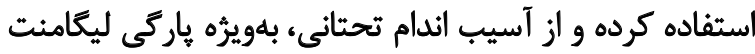
صليبى قدامى دور بماند. براى رسيدن به اين مهارت، بازيكن به توانايى فيزيكى و

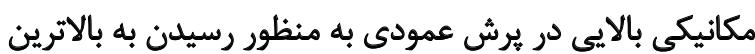

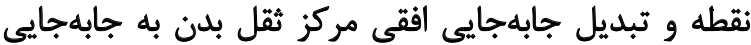

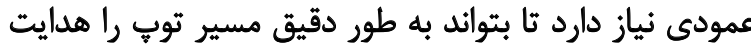

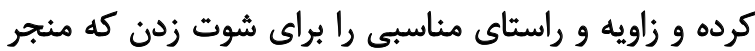

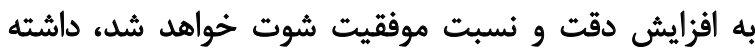

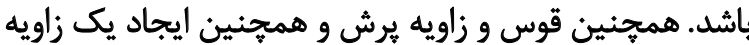

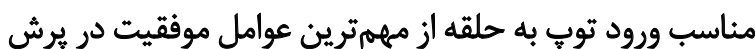

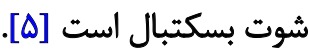

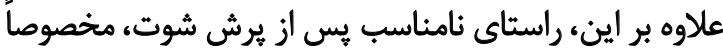

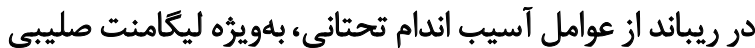

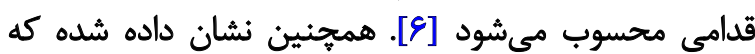

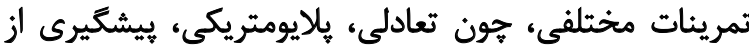

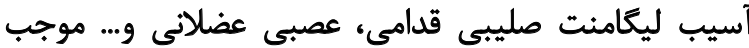

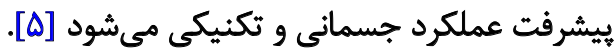

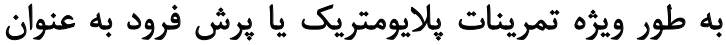

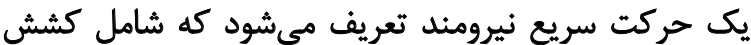

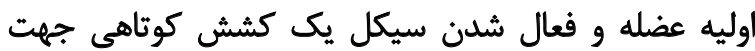




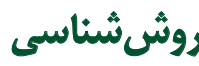

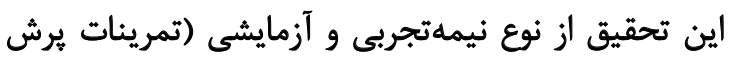

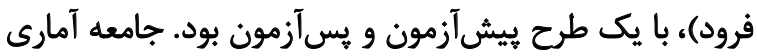

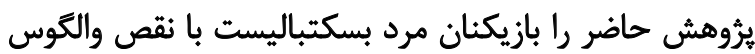

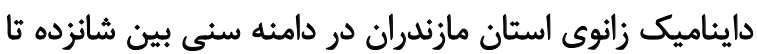

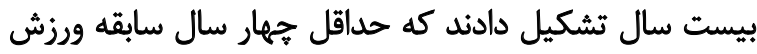

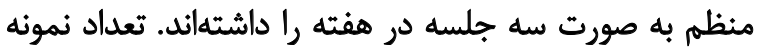

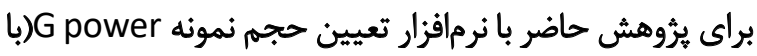

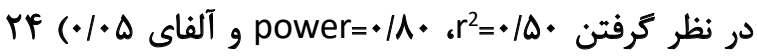

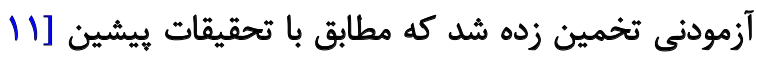

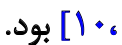

همجنين آزمودنىها به صورت تصادفى در دو تروه آزمايش

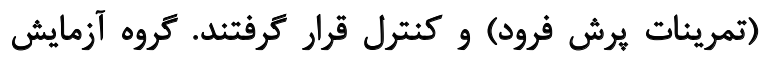

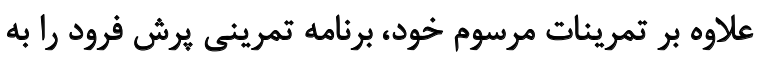

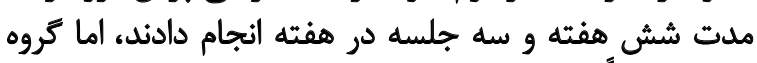

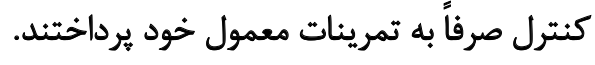

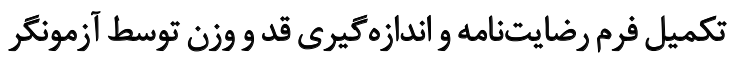

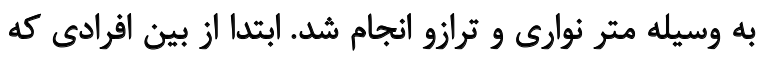

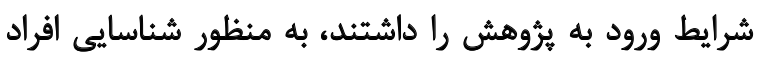

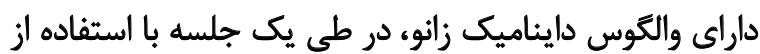

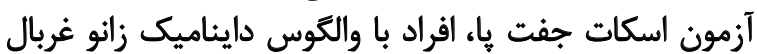

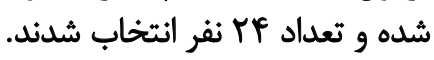
در ادامه مهارت شوت آزمودنىها به كمك آزمون مهارت

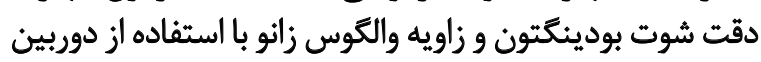

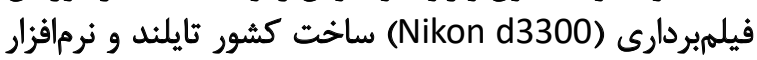

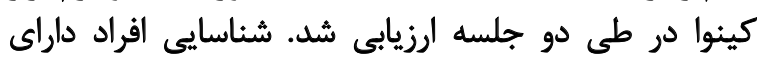

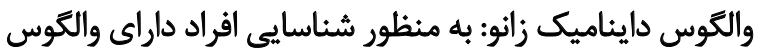

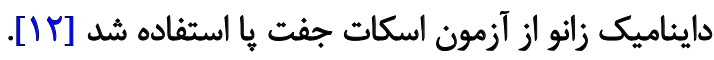

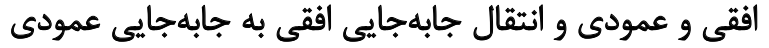

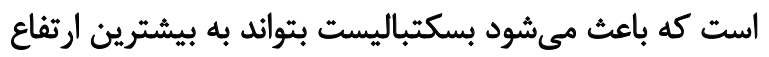

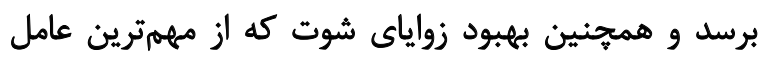

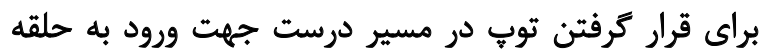

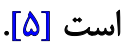
تقريباً در بيشتر تحقيقات در اين زمينه كه تمركز آنها

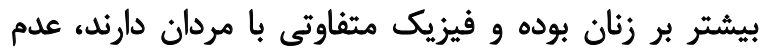

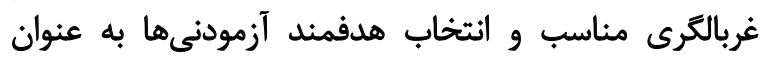

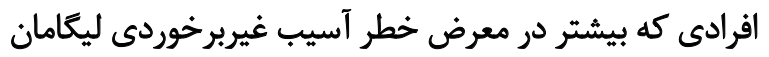

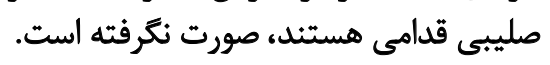

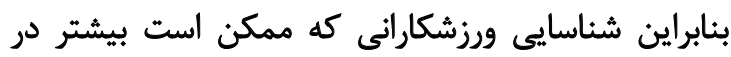

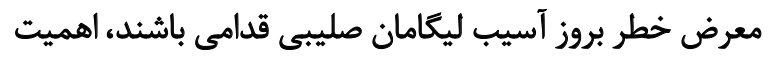

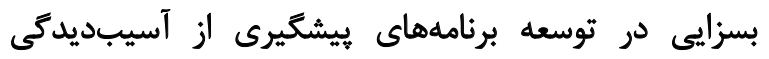

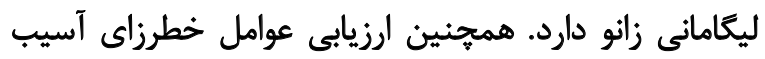

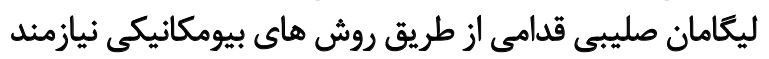

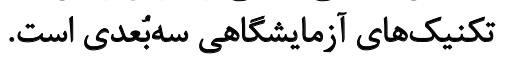

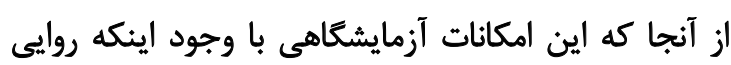

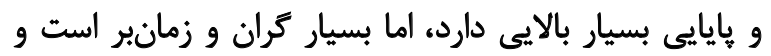

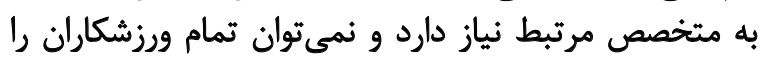

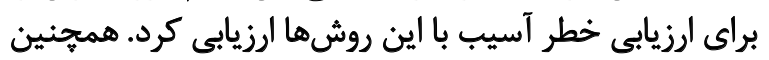

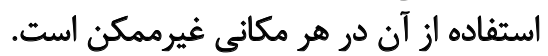

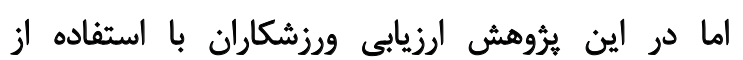

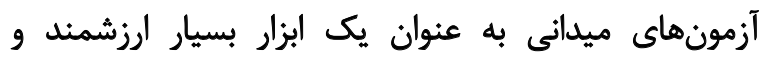

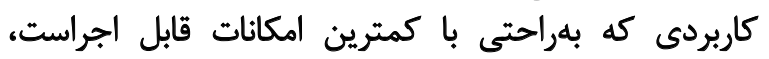

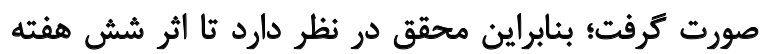

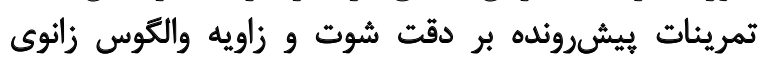

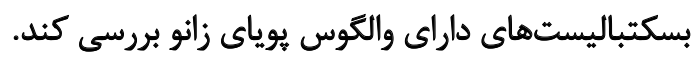

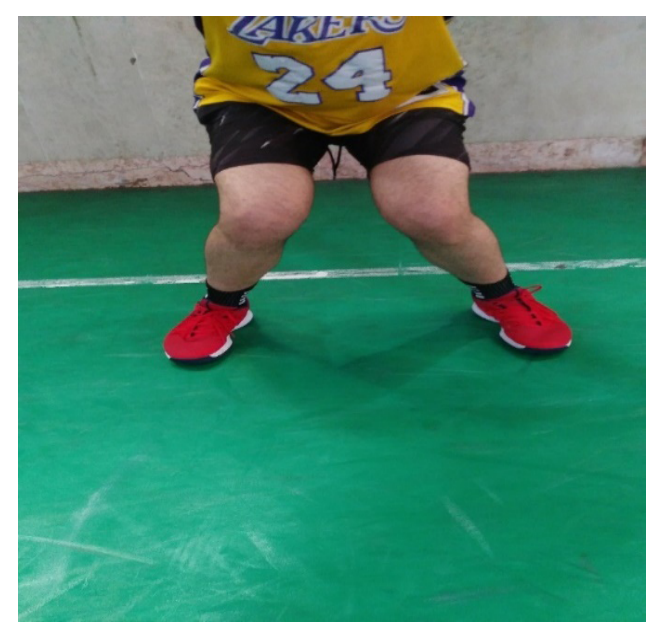




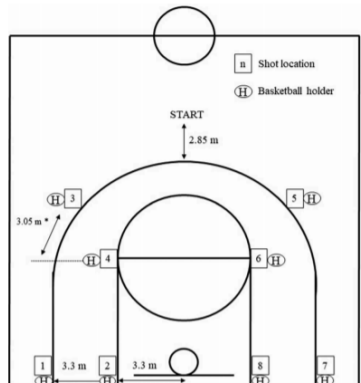

مجله بيومكانيك وزنث

توبَ اول از هشت توبّ موجود در هشت نقطه متفاوت حركت

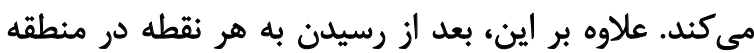

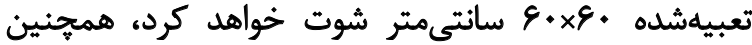

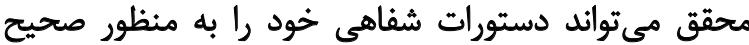

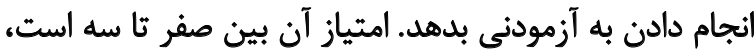

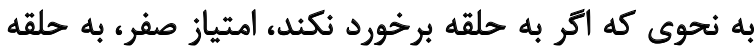

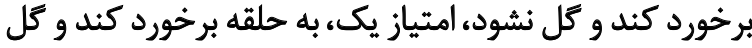

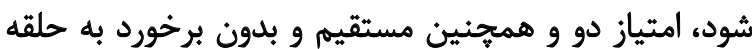

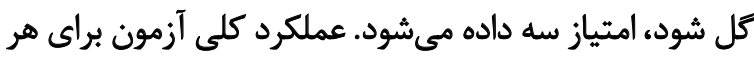

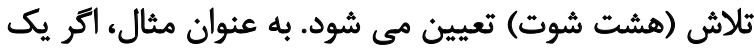

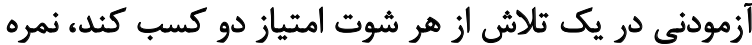

$$
\text { كلى شانزده ثبت مي دشود. }
$$

ثبت امتياز از طريق مشاهده صورت كرفته و همجينين

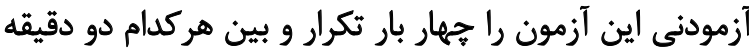

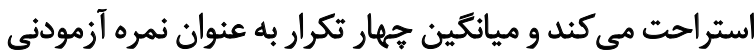

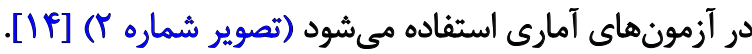

ارزيابى والكوس دايناميك زانو: براى اندازهيرى زاويه

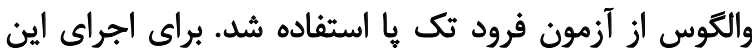

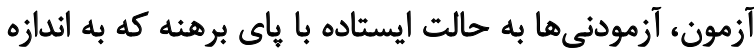

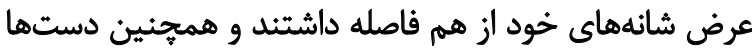

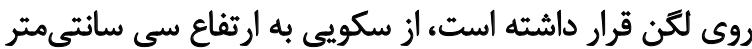

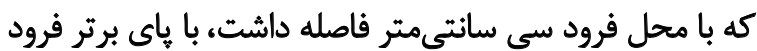

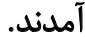

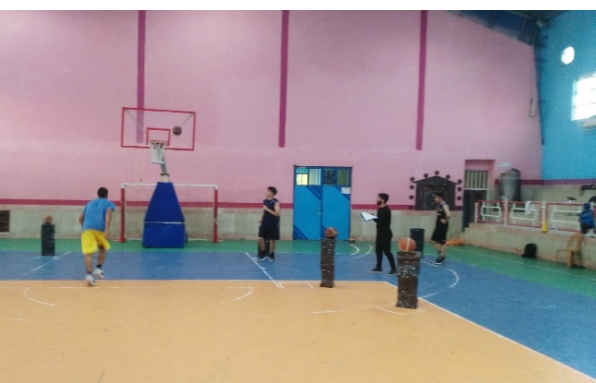

تصوير Y. آزمون مهارت دقت شوت بودينكتون

بر اساس يافتههاى بِل و همكاران تست اسكات بالائ سر ورائ

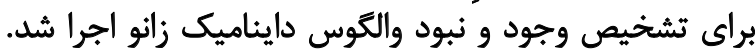

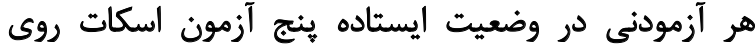

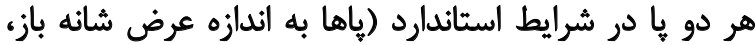

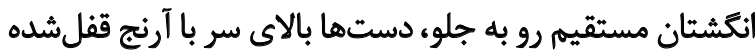

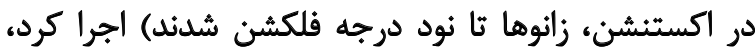

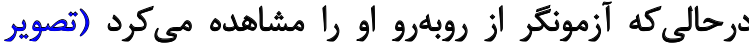

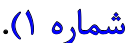

براى به حداقل رساندن اثر يادكيرى اجازه داده نمى شيد كه

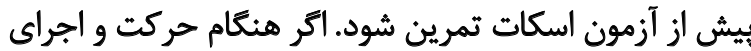

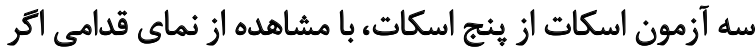

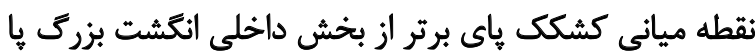

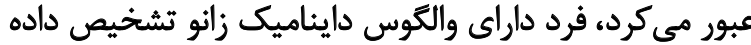

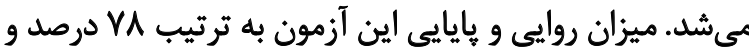

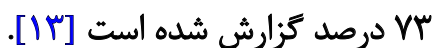

ارزيابى آزمون مهارت شوت بودينكتون (19 + ج): اين آزمون

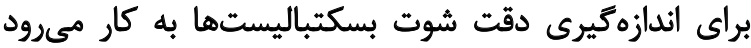

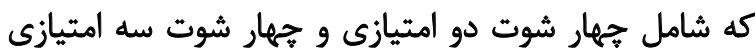

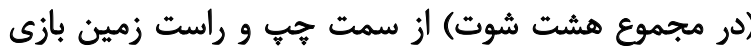

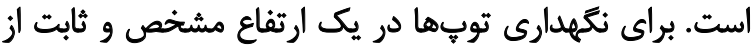
دستكاههاى نكَدارنده به طول يك متر استفاده شد. آزمودنى، آزمون را از نيمه زمين شروع كرده و به سمت 

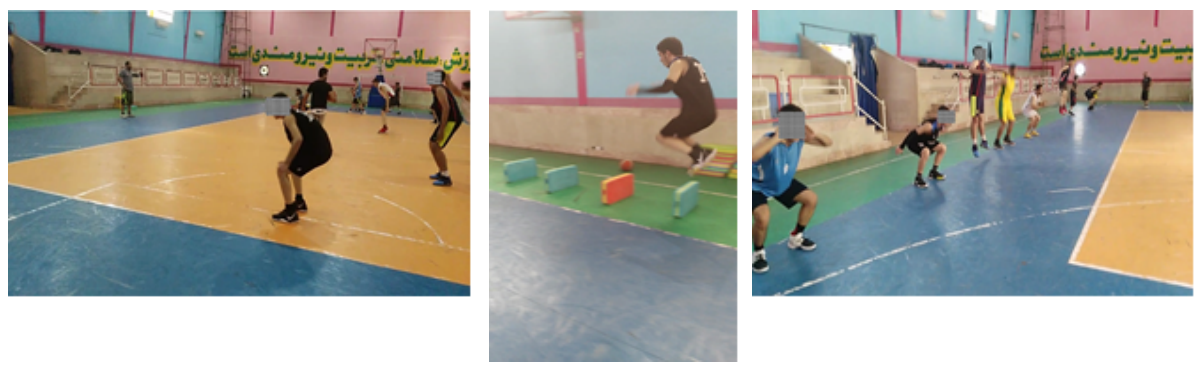

مجله بيومكانيك وزنث

تصوير F. تمريئات

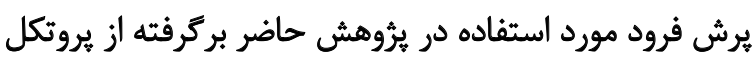

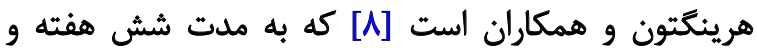

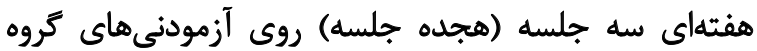

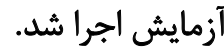

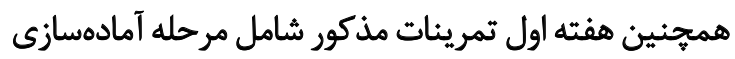

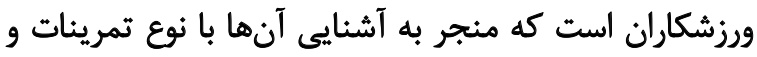

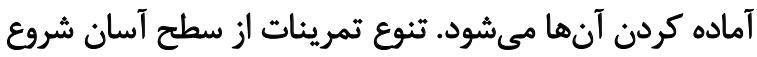

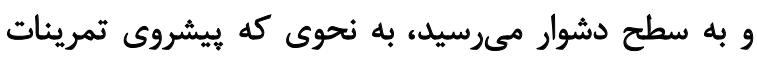

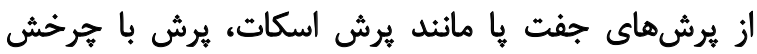

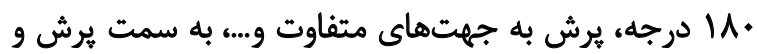

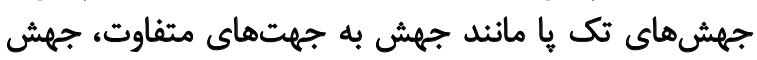

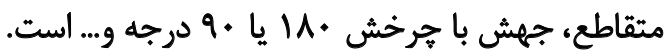

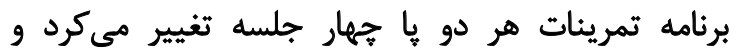

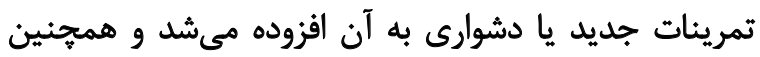

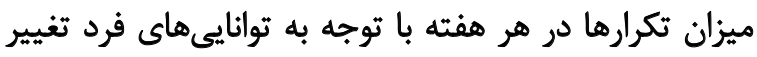

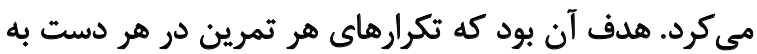
ميزان يرش در هفته يروتكل برد برسد.

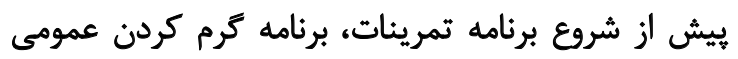

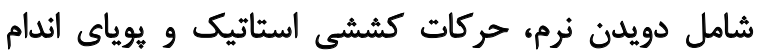

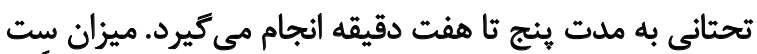

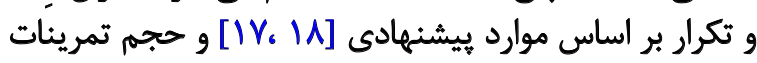

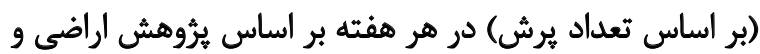

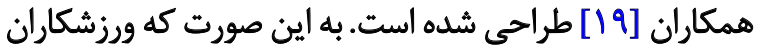

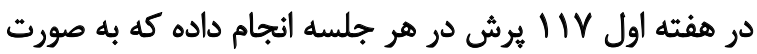

آزمودنى ها هنكام آزمون فعاليتهايي مانئد يرش به به بالا يا

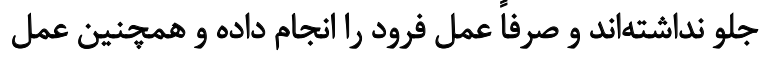

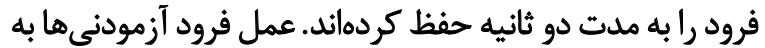

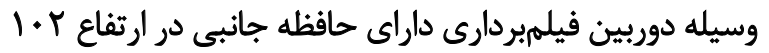

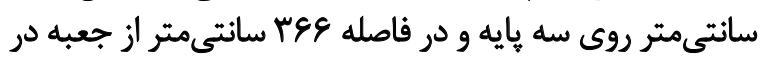

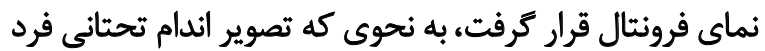

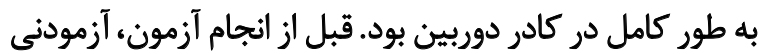

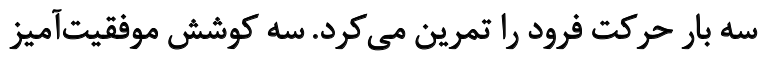

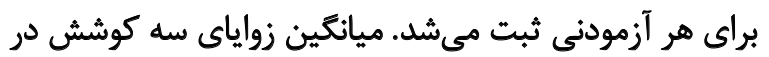

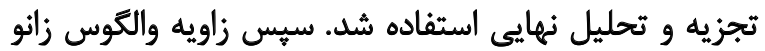

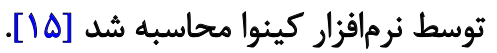

درواقع، با بررسى فريم به فريم در تصويرهاى ويدئويى ترفته

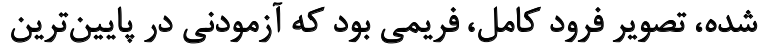

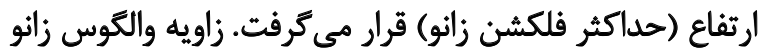

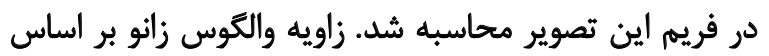

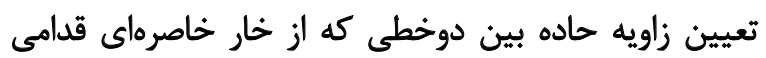

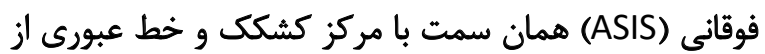

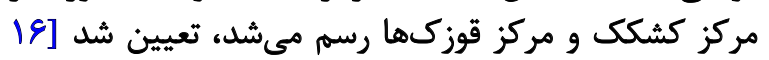

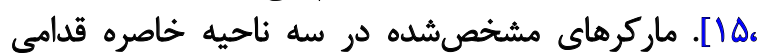

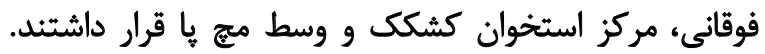

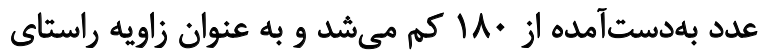

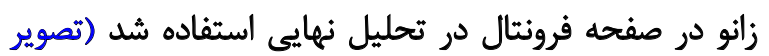

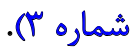
برنامه تمرينى يرش فرود: يروتكل تمرينات بيشرونده

جدول ا. ميانكين و انحراف استاندارد مشخصات فردى و آنترويومتريك آزمودنىها

\begin{tabular}{|c|c|c|c|}
\hline \multirow{2}{*}{$\mathbf{P}$} & \multicolumn{2}{|c|}{ ميانكين||نحراف معيار } & \multirow{2}{*}{ متغير } \\
\hline & كنترل (دوازده نفر) & آزمايش (دوازده نفر) & \\
\hline$\cdot N$ & $\mid V / 9 Y \pm 1 / \Delta$ & $I V / \Delta A \pm I / I V$ & سن (سال) \\
\hline .10 & $19 r / g Y \pm P / P q$ & $191 / \pi T \pm \Delta / \cdot \Lambda$ & قداندىمتر) \\
\hline.$/ r r$ & $A V / M T \pm \Delta / Y q$ & $N E / Q Y \pm T / F T$ & وزن (كيلوكرم) \\
\hline$\cdot / \Delta V$ & $M / \Delta \pm \cdot / E q$ & $T H / N \Delta \pm \cdot|\Lambda|$ & شاخص توده بدنى (كيلوكرم بر متر هربع) \\
\hline
\end{tabular}

مجله بيومكانيك وزنث 
جدول r. آزمون شاييرو ويلك به منظور سنجش طبيعى بودن دادها

\begin{tabular}{|c|c|c|c|c|}
\hline \multicolumn{2}{|c|}{ كروه كتترل (دوازده نفر) } & \multicolumn{2}{|c|}{ تحوه آزمايش (دوازده نفر) } & \multirow{2}{*}{ مثغير } \\
\hline يس آزمون & ييش آزمون & بس آزمون & ي يش آزمون & \\
\hline.$/ 19$ & 年 &.$/ T V$ &.$/ 1$ & شوت بسكتبال \\
\hline.$|+|$ &.$/ T Y$ &.$/ 19$ & .11 & دايناميك والكوس زانو \\
\hline
\end{tabular}

مجله بيومكانيك ولنش

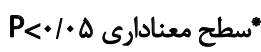

عامل براى قرار گرفتن توب در مسير درست جهت ورود به

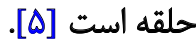

اما تمركز يُوهشهاى يادشده بر افراد سالم و بدون

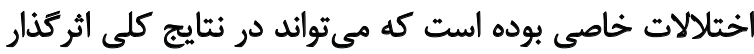

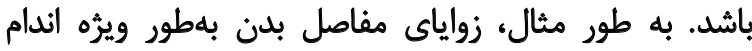

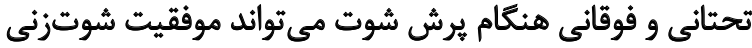

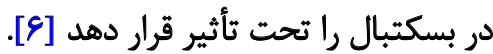
درواقع، والكوس يوياى زانو كه يكى از عوامل آسيبديدكى لئى

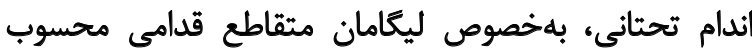

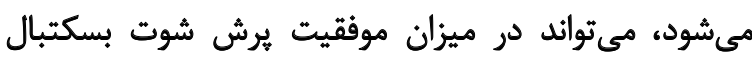

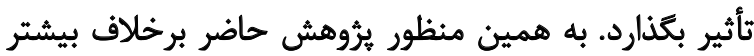
يُروهش ها از افراد داراى والكوس يوياى زئن زانو استفاده كرده است.

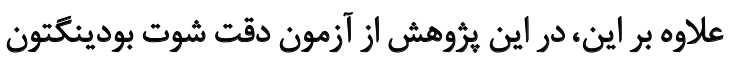

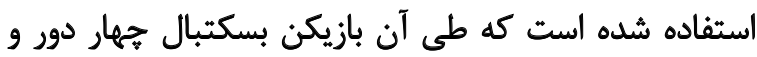

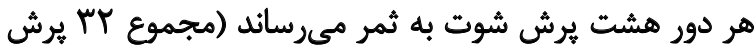

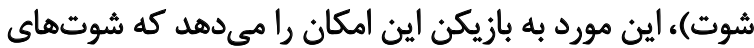

كافى را براي ارزيابى دقيق دقت شوت انجام دهد [1f] در مهارت يرش شوت، هدف رسيدن به بالاترين نقطه بدون

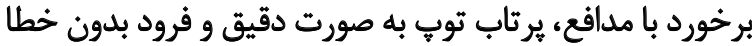

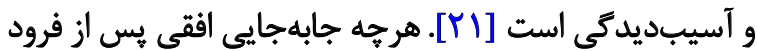

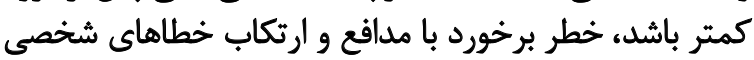

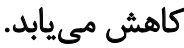

يَيشرونده در هر هفته به تعداد يرش اضافه خواهد شد (تصوير

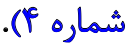

به منظور مقايسه دو كروه آزمايش و كنترل در صورت نرمال

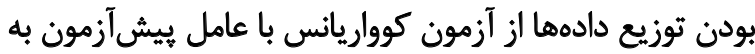
عنوان كووريت استفاده شده است.

نثايج

جدول شماره ا ويزٔحىهاى آنترويومتريك تروه آزمايش

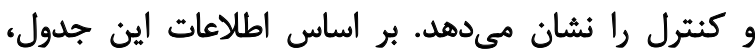

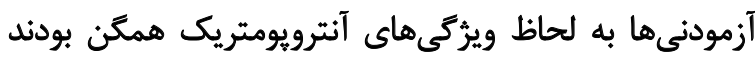

. $(P>\cdot 1 \cdot \Delta)$

حسين محمود و مونيم الوسوفى، در يرؤوهشى اثر تمرينات

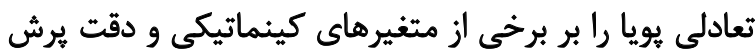

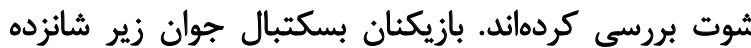

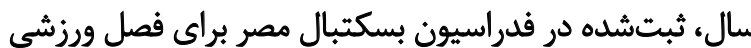

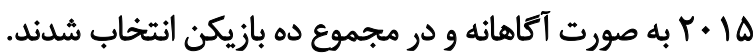

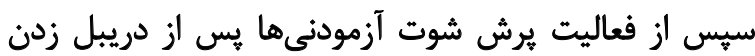

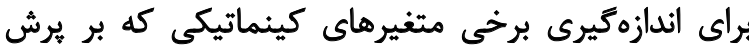
شوت تأثير مى كذارند، فيلم ترفته شدي مئل

نتايج بيانكر بهبود در مسير حركت مركز ثقل براي مسيرهاى جاي

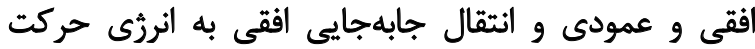

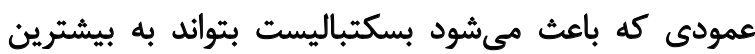

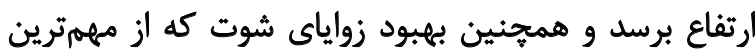

جدول لا. مقايسه متغيرها در يسآزمون بين كروهها

\begin{tabular}{|c|c|c|c|c|c|c|}
\hline مقدار اثر & $\mathbf{P}$ & درجه آزادى & Fسبت F & مياتكين & تحروه & متغير \\
\hline \multirow[t]{2}{*}{.$/ 94$} & \multirow[t]{2}{*}{$.1 .+1$} & \multirow[t]{2}{*}{1} & \multirow[t]{2}{*}{$v \varepsilon / T W$} & & & \multirow[t]{2}{*}{ دقت شوت } \\
\hline & & & & 1.11 & كتترل & \\
\hline \multirow{2}{*}{.$/ 91$} & \multirow{2}{*}{$\%+1$} & \multirow{2}{*}{1} & \multirow{2}{*}{ TE/KGT } & $\mid \theta / 1$ & آزمايش & \multirow{2}{*}{ زاويه والكوس زانو } \\
\hline & & & & W. & كنترل & \\
\hline
\end{tabular}

مجله بيومكانيكوزنش 


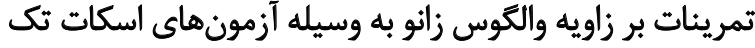
ياء تك يا و جفت يا محاسبه شد.

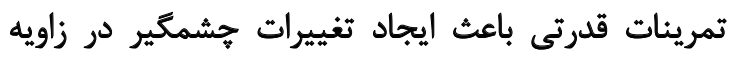

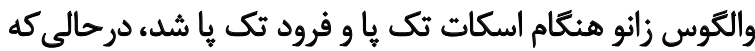

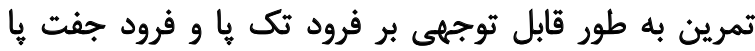

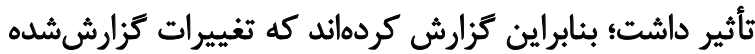

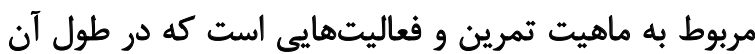

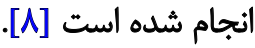

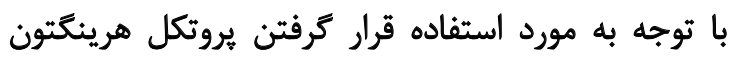

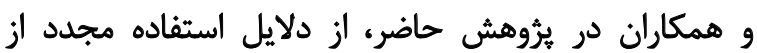

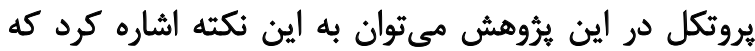

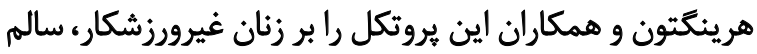

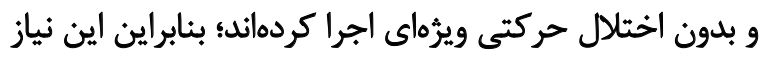

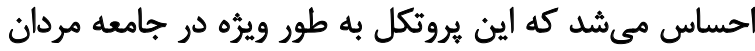

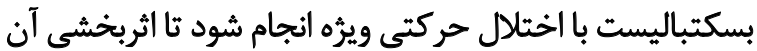
مورد آزمون قرار كيرد.

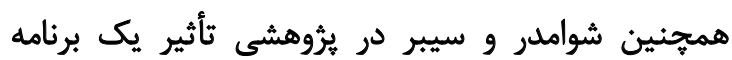

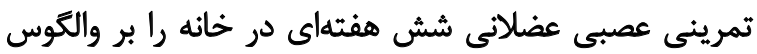

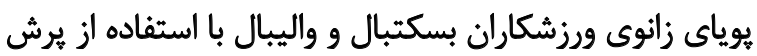

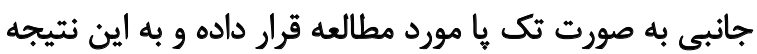

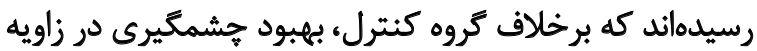

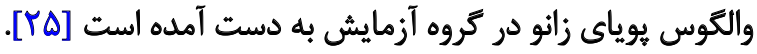

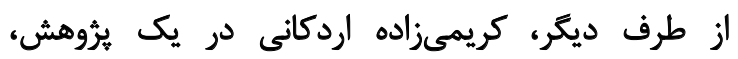

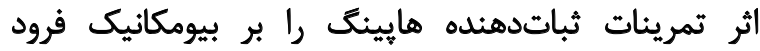

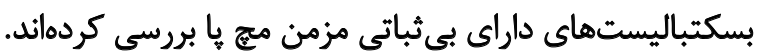

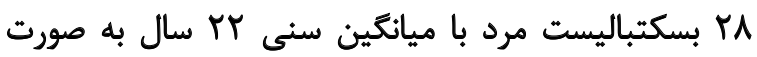

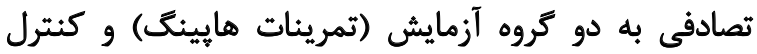

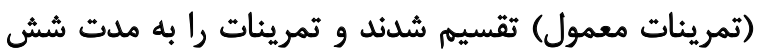

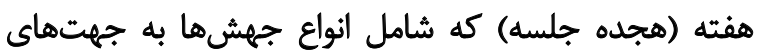

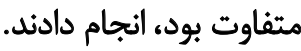

كينتيك و كينماتيك اندام تحتانى در يك فعاليت و نيز

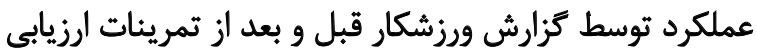

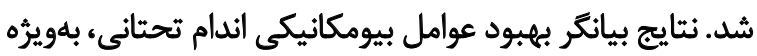

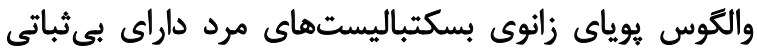

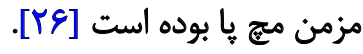

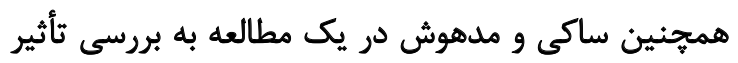

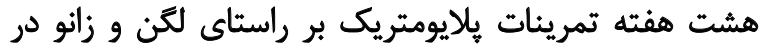

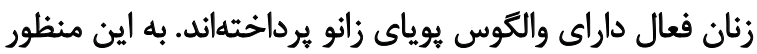

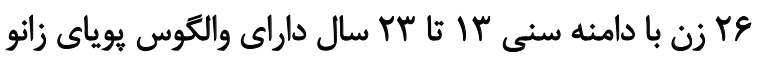

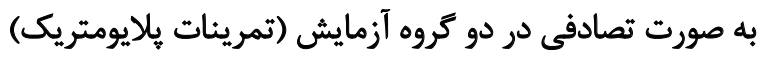
و كنترل (تمرينات معمول) قرار كرفتند.

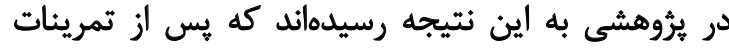

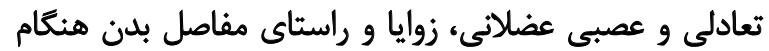

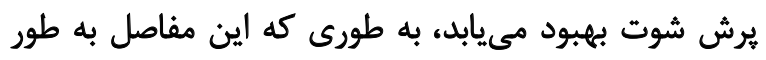

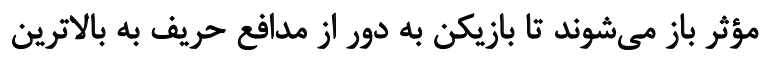

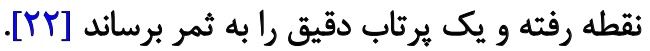

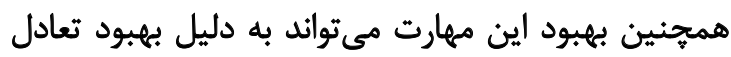

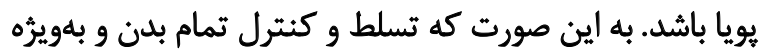

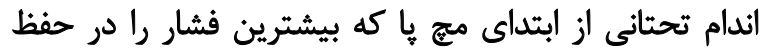

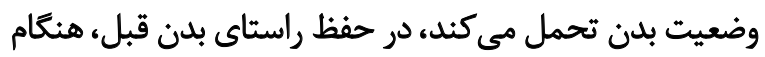
و و بعد از برش شوت مؤثر است.

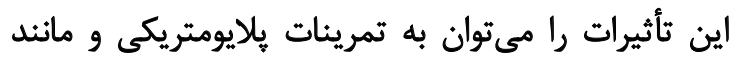

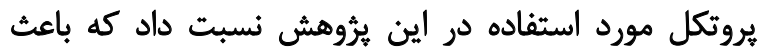

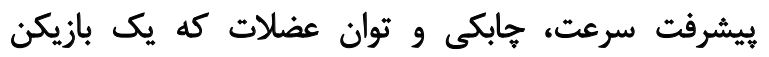

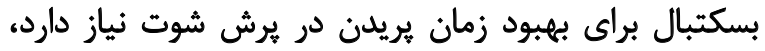
خواهد شد [ه].

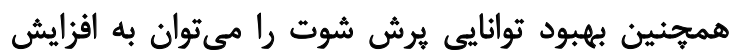

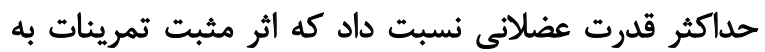

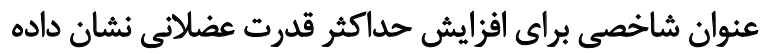

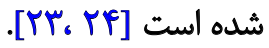

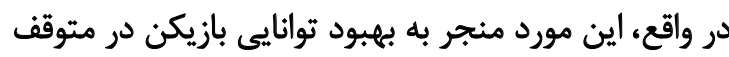

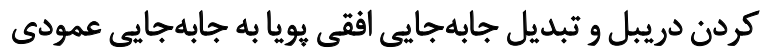

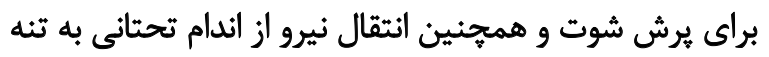

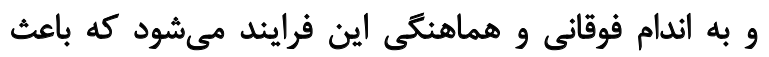

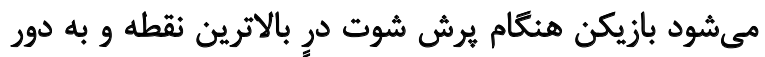

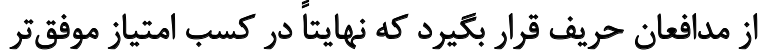

خواهد بود [ه].

از طرف ديكر، مطالعه حاضر نشان داد كه شش هفته تمرين

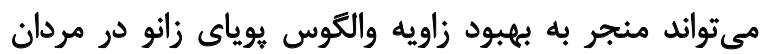

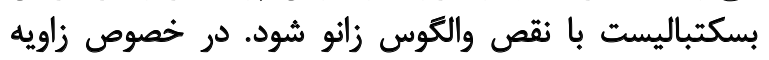

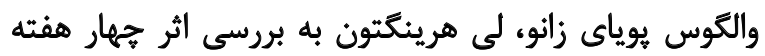

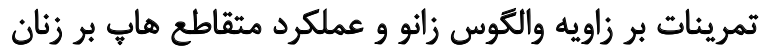

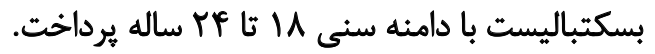

محقق به دنبال بيى بردن اين بوده است كه آيا تمرينات

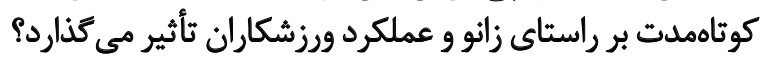

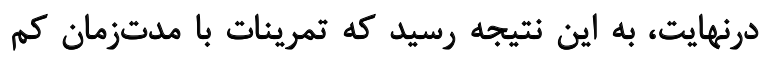

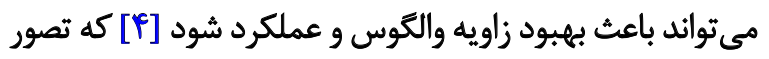

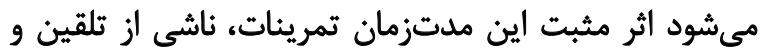
بهبود عملكرد عصبى عضلانى باشد.

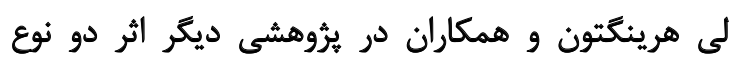

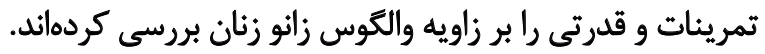
تمرينات به مدت شش هفته (حداقل يانزده جلسه) انجام و اثر زئر 
نكته اشاره كرد كه در طول تمرينات، آزمودنىها دستور العمل

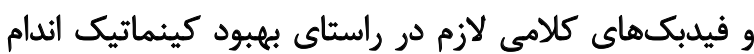

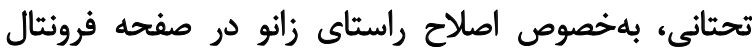

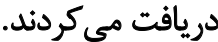

به عبارت ديكر، مي توان به اين نتيجه رسيد كه آزمودنى بها

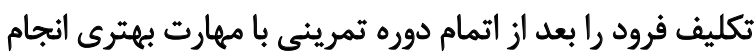

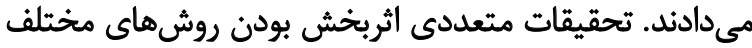

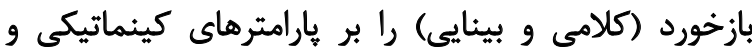

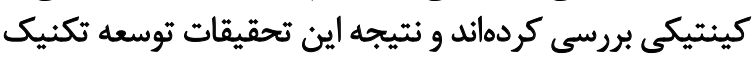

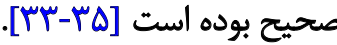

يك نقص عمده بيشتر تحقيقات كذشته استفاده از بازخورد

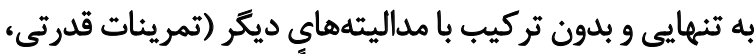

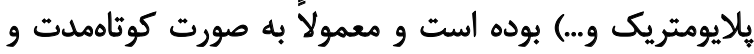

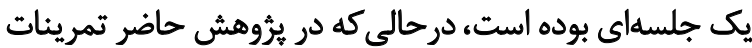

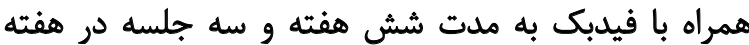

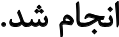

\section{نتيجلكَيرى نهايي}

نتايج يرؤش حاضر شواهدى مبنى بر اثر مثبت تمرينات

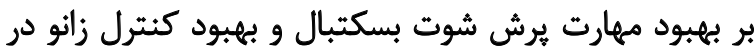

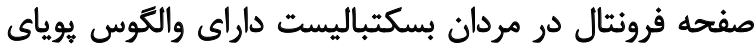

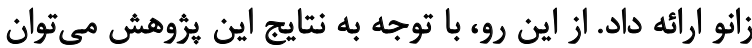

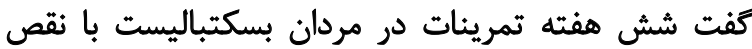

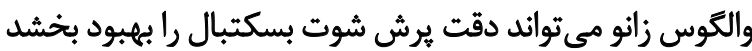

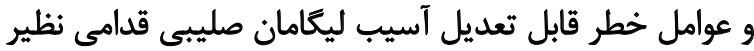

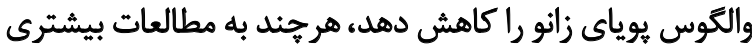
در اين زمينه نياز است.

بيشنهاد مي شود محققان و مربيان براى طراحى آزمايشات

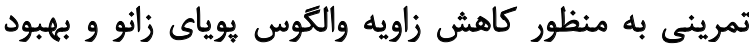

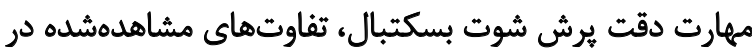

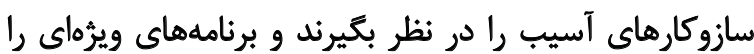

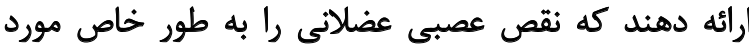

توجه قرار دهد. - اند

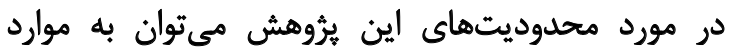

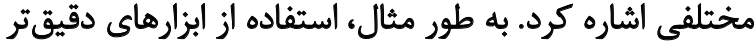

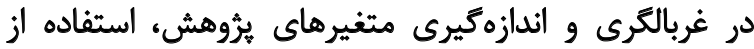

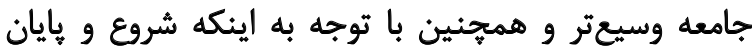

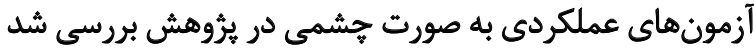

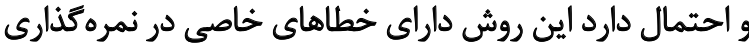

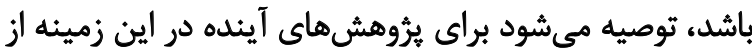
دستكاههاى فوتوفينيش استفاده شود.
آزمودنى هايي وارد مطالعه شدهاند كه زاويه والكوس بيشتر

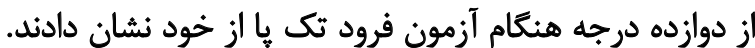

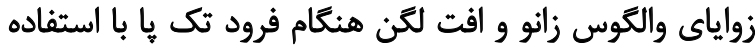

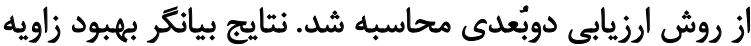

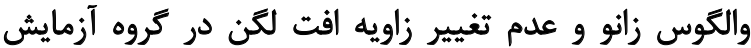

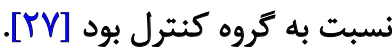

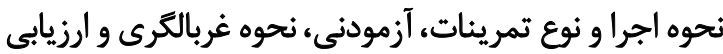

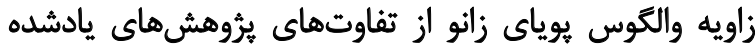

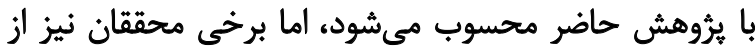

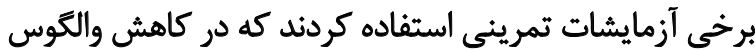

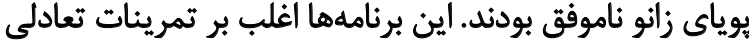

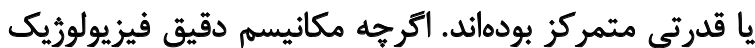

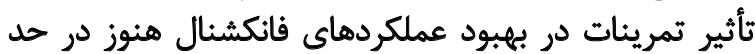

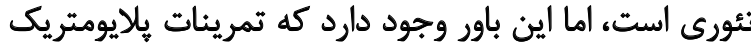

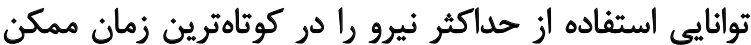

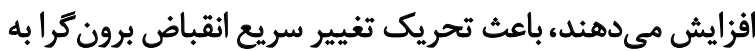

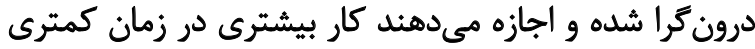

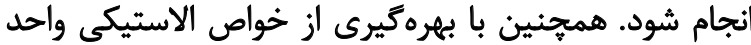

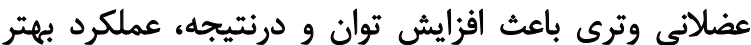

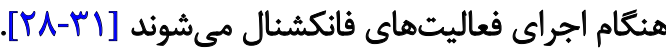
آر جه مقايسه اين تحقيقات بهطور كامل ممكن نيست، زيرا

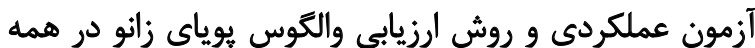

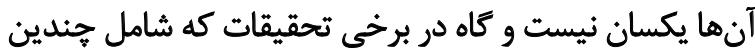

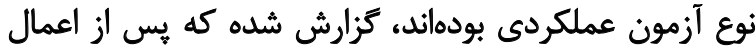

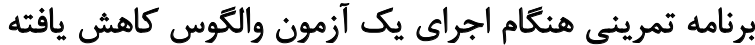

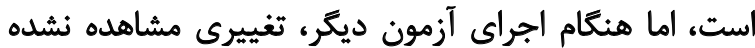

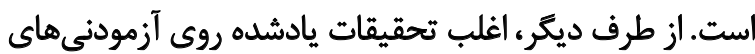

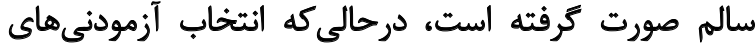

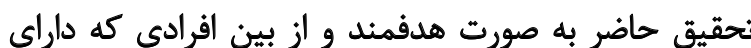
والكوس يوياى زانو بودند، انجام شد. هدفي

تاكنون برنامههاى تمرينى مختلفى براى كاهش آسيبهاى ترئي

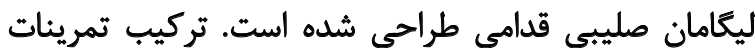

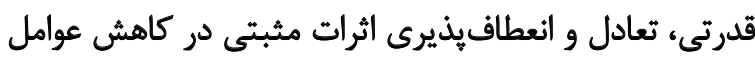
بيومكانيكى خطرساز آسيب ليكامان صليبى قدامى داني داشتهاني داند

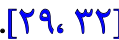

اتر جها ميزان اثربخشى هر جزء اين برنامهها از نظر درصد

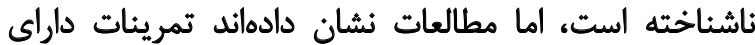

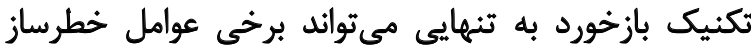

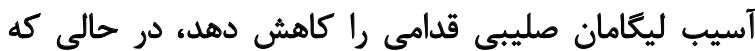

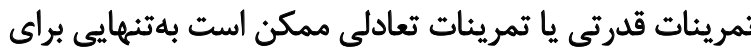

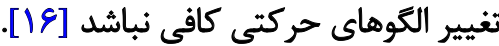

از دلايل احتمالى اثربخشى مطالعه حاضر مىتوان به اين 


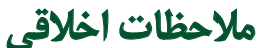

بيروى از اصول اخلاق يثوهش

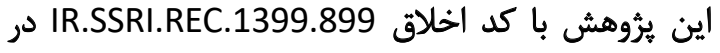
يروهشاء تربيتبدنى و علوم ورزشى تصويب شداف

$$
\text { مامى مالى }
$$

اين مقاله از باياننامه كارشناسى ارشد سعيد قبادى ترُّاد، در

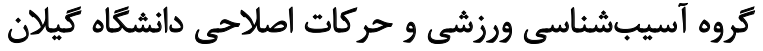
استخراج شده است. - است ورزش

$$
\text { مشاركت نويسند مَّان }
$$

تمام نويسندكان در طراحى، اجرا و نكارش همه بخش هاي ئروهش حاضر مشاركت داشتهاند.

$$
\text { تعارض مناقع }
$$

طبق نظر نويسندكان اين ثُروهش تعارض منافع ندارد. 


\section{Refrences}

[1] Letafatkar A, Rajabi R, Tekamejani EE, Minoonejad H. Effects of perturbation training on knee flexion angle and quadriceps to hamstring cocontraction of female athletes with quadriceps dominance deficit: Pre-post intervention study. Knee. 2015; 22(3):230-6. [DOI:10.1016/j. knee.2015.02.001] [PMID]

[2] Boden BP, Torg JS, Knowles SB, Hewett TE. Video analysis of anterior cruciate ligament injury:Abnormalities in hip and ankle kinematics. Am J Sports Med. 2009; 37(2):252-9. [DOI:10.1177/0363546508328107] [PMID]

[3] Krosshaug T, Nakamae A, Boden BP, Engebretsen L, Smith G, Slauterbeck JR, et al. Mechanisms of anterior cruciate ligament injury in basketball: Video analysis of 39 cases. Am J Sports Med. 2007; 35(3):35967. [DOI:10.1177/0363546506293899] [PMID]

[4] Herrington L. The effects of 4 weeks of jump training on landing knee valgus and crossover hop performance in female basketball players. J Strength Cond Res. 2010; 24(12):3427-32. [DOI:10.1519/ JSC.0b013e3181c1fcd8] [PMID]

[5] Mahmoud MH, Elseuofy AAM. The effect of dynamics balance exercises on some kinematics variables and jump shoot accuracy for young basketball players [Internet]. 2010 [Updated 2010 July 15]. Available from: https://www.researchgate.net/profile/MahmoudHoussain/publication/320616580_06/data/59f0b8110f7e9beabfca 3c23/06.pdf

[6] Struzik A, Pietraszewski B, Zawadzki J. Biomechanical analysis of the jump shot in basketball. J Hum Kinet. 2014; 42(1):73-9. [DOI:10.2478/ hukin-2014-0062] [PMID] [PMCID]

[7] Myer GD, Ford KR, Palumbo JP, Hewett TE. Neuromuscular training improves performance and lower-extremity biomechanics in female athletes. J Strength Cond Res. 2005; 19(1):51-60. [DOI:10.1519/00124278-200502000-00010] [PMID]

[8] Herrington L, Munro A, Comfort P. A preliminary study into the effect of jumping-landing training and strength training on frontal plane projection angle. Man Ther. 2015; 20(5):680-5. [DOI:10.1016/j. math.2015.04.009] [PMID]

[9] Ahmed TAE. Improving musculoskeletal fitness and the performance enhancement of basketball skills through neuromuscular training program. J Hum Sport Exerc. 2015; 10(3):795-804. [DOI:10.14198/ jhse.2015.103.05]

[10] Keshavarz L, Letafatkar A, Hadadnezhad M. [Effect of eight weeks of jump-landing exercise on feedforward and feedback activation of selected trunk and lower extremity muscles and lower extremity performance in active females (Persian)]. Med J Tabriz Univ Med Sci. 2018; 40(5):72-82. https://mj.tbzmed.ac.ir/Article/23962

[11] Poorkiani M, Letafatkar A, Hadadnejad M, Shojaedin SS. [Effectiveness of eight weeks of progressive jump-landing exercises on performance and dynamic balance of young footballers at the risk of anterior cruciat ligament injury (Persian)]. Sci J Rehabil Med. 2018; 7(3):59-68. [DOI:10.22037/JRM.2018.110673.1446]

[12] Bell DR, Vesci BJ, Distefano L, Guskiewicz KM, Hirth CJ, Padua DA. Muscle activity and flexibility in individuals with medial knee displacement during the overhead squat. Athl Train Sport Health Care. 2012; 4(3):117-25. [DOI:10.3928/19425864-20110817-03]

[13] Powers CM. The influence of abnormal hip mechanics on knee injury: A biomechanical perspective. J Orthop Sports Phys Ther. 2010; 40(2):42-51. [DOI:10.2519/jospt.2010.3337] [PMID]
[14] Boddington BJ, Cripps AJ, Scanlan AT, Spiteri T. The validity and reliability of the basketball jump shooting accuracy test. J Sports Sci. 2019; 37(14):1648-54. [DOI:10.1080/02640414.2019.1582138] [PMID]

[15] Herrington L, Munro A. Drop jump landing knee valgus angle; Normative data in a physically active population. Phys Ther Sport. 2010; 11(2):56-9. [DOI:10.1016/j.ptsp.2009.11.004] [PMID]

[16] Koorosh-Fard N, Ali-Zadeh MH, Rajabi R, Shirzad E. [Effect of feedback corrective exercise on knee valgus and electromyographic activity of lower limb muscles in single leg squat (Persian)]. Arch Rehabil. 2015; 16(2):138-47. http://rehabilitationj.uswr.ac.ir/article1-1547-en.html

[17] Alver BA, Sell K, Deuster PA. NSCA's essentials of tactical strength and conditioning. Champaign, IL: Human Kinetics; 2017. https:// books.google.com/books?id=GHcBDgAAQBAJ\&printsec=frontcover \&dq

[18] Piper TJ, Erdmann LD. Journal C. A 4-step plyometric program. Strength Cond J. 1998; 20(6):72-3. https://journals.Iww.com/nscascj/Citation/1998/12000/A_4_Step_Plyometric_Program.13.aspx

[19] Arazi H, Asadi A. The effect of aquatic and land plyometric training on strength, sprint, and balance in young basketball players. Revistas J Hum Sport Exerc. 2011; 6(1):101-11. [DOI:10.4100/jhse.2011.61.12]

[20] Ilham I, Muhammad Ali M, David Iqroni D. The plyometric training on free throw shooting ability and skills in basketball. J Crit Rev. 2020; 7(14):808-14. [DOI:10.31838/jcr.07.14.144]

[21] Elsoufy AAE. Movement science traditional and contemporary Cairo: DarrFekrah; 2012.

[22] Aalhawary R. The effect of dynamic balance exercises on certain kinematic variables and jump shoot accuracy among female basketball players. J Phys Educ Health. 2019; 8(14):41-8. [DOI:10.5281/ zenodo.3746167]

[23] Asadi A, Arazi H. Effects of high-intensity plyometric training on dy namic balance, agility, vertical jump and sprint performance in young male basketball players. J Sport Health Res. 2012; 4(1):35-44. https:// www.researchgate.net/publication/266318427

[24] Hadi P, Doewes M, Riyadi S. The influence of low intensity-high intensity plyometric training and hand-eye coordination on jump shoot ability in basketball players of bhinneka solo club: Randomized control trial. Budapest Int Res Crit Linguist Educ J. 2020; 3(1):514-22. [DOI:10.33258/birle.v3i1.847]

[25] Schwameder $\mathrm{H}$. Effect of a neuromuscular home training program on Dynamic Knee Valgus (DKV) in lateral single-leg landings. ISBS Proc Arch. 2020; 38(1):186. https://commons.nmu.edu/isbs/vol38/ iss1/186/

[26] Karimizadeh Ardakani M, Wikstrom EA, Minoonejad H, Rajabi R Sharifnezhad A. Hop-stabilization training and landing biomechanics in athletes with chronic ankle instability: A randomized controlled trial. J Athl Train. 2019; 54(12):1296-303. [DOI:10.4085/1062-6050550-17] [PMID] [PMCID]

[27] Saki F, Madhosh M. [Effect of eight weeks plyometric training on pelvic and knee alignment in female with dynamic knee valgus (Persian)]. J Res Sport Rehabil. 2019; 6(12):21-9. [DOI:10.22084/ RSR.2019.17644.1415]

[28] Chmielewski TL, Myer GD, Kauffman D, Tillman SM. Plyometric exercise in the rehabilitation of athletes: Physiological responses and clinical application. J Orthop Sports Phys Ther. 2006; 36(5):308-19. [DOI:10.2519/jospt.2006.2013] [PMID] 
[29] Distefano L, Blackburn JT, Marshall SW, Guskiewicz KM, Garrett WE, Padua DA. Effects of an age-specific anterior cruciate ligament injury prevention program on lower extremity biomechanics in children. Am J Sports Med. 2011; 39(5):949-57. [DOI:10.1177/0363546510392015] [PMID]

[30] Myer GD, Ford KR, Hewett TE. Methodological approaches and rationale for training to prevent anterior cruciate ligament injuries in female athletes. Scand J Med Sci Sports. 2004; 14(5):275-85. [DOI:10.1111/ j.1600-0838.2004.00410.x] [PMID]

[31] Pollard CD, Sigward SM, Ota S, Langford K, Powers CM. The influence of in-season injury prevention training on lower-extremity kinematics during landing in female soccer players. Clin J Sport Med. 2006; 16(3):223-7. [DOI:10.1097/00042752-200605000-00006] [PMID]

[32] McCann R, Cortes N, Van Lunen B, Greska E, Ringleb S, Onate J. Neuromuscular changes following an injury prevention program for $\mathrm{ACL}$ injuries. Int J Athl Ther Train. 2011; 16(4):16-20. [DOI:10.1123/ijatt.16.4.16]

[33] Aagaard P. The use of eccentric strength training to enhance maximal muscle strength, explosive force (RDF) and muscular power-consequences for athletic performance. Open Sports Sci J. 2010; 3:52-5. [DOI: 10.2174/1875399X010030100052]

[34] Rimmer E, Sleivert G. Effects of a plyometrics intervention program on sprint performance. J Strength Cond Res. 2000; 14(3):295-301. [DOI:10.1519/00124278-200008000-00009]

[35] Stickler L, Goehring M, Kinne B. The impact of hip strengthening and/ or neuromuscular control on frontal plane knee kinematics and kinetics in females: A systematic review. Phys Ther Rev. 2016; 21(1):10-6. [DOI:1 0.1080/10833196.2016.1214357] 
This Page Intentionally Left Blank 